\title{
Altered Distributions of Bone Tissue Mineral and Collagen Properties in Women with Fragility Fractures
}

\author{
Zhen Xiang Wang ${ }^{1}$, Ashley A. Lloyd ${ }^{1}$, Jayme C. Burket ${ }^{2}$, Samuel Gourion-Arsiquaud ${ }^{3}$ \\ and Eve Donnelly 1,2 \\ ${ }^{1}$ Department of Materials Science and Engineering, Cornell University, Ithaca, NY \\ ${ }^{2}$ Hospital for Special Surgery, New York, NY \\ ${ }^{3}$ TRI Princeton, Princeton, NJ
}

Funding for this work:

ZXW: Cornell Engineering Learning Initiatives

AAL: National Science Foundation Graduate Research Fellowship Program, Grant No. NSF DGE-1144153

ED: American Society for Bone and Mineral Research Junior Faculty Osteoporosis

Research Award

Contact information for corresponding author:

Eve Donnelly, Ph.D.

Assistant Professor

Department of Materials Science and Engineering

Cornell University

227 Bard Hall

Ithaca, NY 14853

Tel: $607-255-1067$

Fax: $\underline{607-255-2365}$

Email: eve.donnelly@cornell.edu 


\section{ABSTRACT}

Heterogeneity of bone tissue properties is emerging as a potential indicator of altered bone quality in pathologic tissue. The objective of this study was to compare the distributions of tissue properties in women with and without histories of fragility fractures using Fourier transform infrared (FTIR) imaging. We extended a prior study that examined the relationship of the mean FTIR properties to fracture risk by analyzing in detail the widths and the tails of the distributions of FTIR properties in biopsies from fracture and non-fracture cohorts. The mineral and matrix properties of cortical and trabecular iliac crest tissue were compared in biopsies from women with a history of fragility fracture $(+\mathrm{Fx} ; \mathrm{n}=21$, age mean $54 \pm \mathrm{SD} 15 \mathrm{y})$ and with no history of fragility fracture $(-\mathrm{Fx} ; \mathrm{n}=12$, age $57 \pm 5 \mathrm{y})$. A subset of the patients included in the $-\mathrm{Fx}$ group were taking estrogen-plus-progestin hormone replacement therapy (HRT) $(-\mathrm{Fx}+\mathrm{HRT}$ $\mathrm{n}=8$, age: $58 \pm 5 \mathrm{y}$ ) and were analyzed separately from patients with no history of HRT (Fx-HRT n=4, age: $56 \pm 7$ y). When the FTIR parameter mean values were examined by treatment group, the trabecular tissue of $-\mathrm{Fx}-\mathrm{HRT}$ patients had a lower mineral:matrix ratio (M:M) and collagen maturity (XLR) than that of $-\mathrm{Fx}+\mathrm{HRT}$ patients $(-22 \% \mathrm{M}: \mathrm{M}$, 18\% XLR) and +Fx patients (-17\% M:M, -18\% XLR). Across multiple FTIR parameters, tissue from the $-\mathrm{Fx}-\mathrm{HRT}$ group had smaller low-tail $\left(5^{\text {th }}\right.$ percentile $)$ values than that from the $-\mathrm{Fx}+\mathrm{HRT}$ or $+\mathrm{Fx}$ groups. In trabecular collagen maturity and crystallinity (XST), the -Fx-HRT group had smaller low-tail values than those in the $-\mathrm{Fx}+\mathrm{HRT}$ group $(-16 \%$ XLR, $-5 \%$ XST) and the +Fx group (-17\% XLR, -7\% XST). The relatively low values of trabecular mineral:matrix ratio and collagen maturity and smaller low-tail values of collagen maturity and crystallinity observed in the $-\mathrm{Fx}-\mathrm{HRT}$ group are characteristic of 
younger tissue. Taken together, our data suggest that the presence of newly formed tissue that includes small/imperfect crystals and immature crosslinks, as well as moderately mature tissue, is an important characteristic of healthy, fracture-resistant bone. Finally, the larger mean and low-tail values of mineral:matrix ratio and collagen maturity noted in our $-\mathrm{Fx}+\mathrm{HRT}$ vs. $-\mathrm{Fx}-\mathrm{HRT}$ biopsies are consistent with greater tissue age and greater BMD arising from decreased osteoclastic resorption in HRT-treated patients.

Keywords: bone; osteoporosis; fracture; bone quality; material properties; mineral; collagen; Fourier transform infrared imaging; hormone replacement therapy 


\section{INTRODUCTION}

Fragility fracture risk depends on bone quantity, which is quantified clinically by areal bone mineral density (BMD), and bone quality, which encompasses geometric, microarchitectural, and material factors that contribute to whole-bone fracture resistance. Large epidemiologic studies have demonstrated that variation in BMD accounts for approximately $60 \%$ of fragility fracture risk $[1,2]$, suggesting that the significant remaining proportion of fracture risk not explained by BMD may be explained by variations in bone quality. Factors that contribute to bone quality span multiple levels of bone structural hierarchy and include cancellous microarchitecture, microdamage density and morphology, tissue mineralization, and collagen crosslinking [3].

Fourier transform infrared (FTIR) imaging is capable of characterizing both the organic and the inorganic components of bone tissue in a spatially resolved fashion, and its outcomes are indices of bone quality that have been shown to account for fragility fracture incidence not predicted by BMD [4]. The following FTIR parameters, reviewed in detail elsewhere [5], have been defined and validated to quantify bone mineral and collagen properties: the mineral:matrix ratio, which measures bone mineral content [6]; the carbonate:phosphate ratio, which reflects the extent of carbonate substitution in the hydroxyapatite crystal lattice [7]; the collagen maturity (XLR), which indicates the ratio of nonreducible to reducible enzymatic collagen crosslinking [8]; and the crystallinity (XST), which is related to the mineral crystal size and perfection [9]. A prior study comparing bone tissue composition in women with and without fragility fractures using FTIR imaging identified that the mean values of several FTIR parameters-including the mineral:matrix ratio and collagen maturity in cortical regions as well as the collagen 
maturity and crystallinity in trabecular regions-were statistically associated with fragility fracture risk independently of BMD [4]. However, the cortical and trabecular regions do not exhibit a single homogeneous composition; rather, they are characterized by a heterogeneous distribution of compositional properties.

Spatial variation in bone tissue properties, i.e., heterogeneity, is emerging as a potential indicator of bone quality in healthy and pathologic tissue [10-13]. A narrower distribution of tissue properties at the micrometer scale may contribute to increased fracture risk through a reduction in the tissue's intrinsic toughening mechanisms [14], as has been demonstrated at the $\sim 10 \mathrm{~nm}$ length scale [12]. Although several studies have noted differences in the distributions of tissue material properties in patients with and without histories of fragility fractures, their results diverge concerning which group exhibits greater heterogeneity. In a quantitative backscattered electron imaging (qBEI) analysis of bone tissue from patients with and without fractures, the mineralization distributions of iliac crest trabeculae from patients with fractures had smaller coefficients of variation than those of patients without fractures [15]. Femoral neck tissue of patients with fragility fractures characterized with FTIR imaging had narrower distributions of mineral:matrix ratio and carbonate:phosphate ratio but wider distributions of crystallinity compared to that of fracture-free cadaveric controls [16]. Finally, a microradiograpic study of the femoral neck showed that the heterogeneity of mineralization was greater in patients with a history of fragility fracture vs. non-fracture controls [17]. In summary, consistent trends in tissue heterogeneity with fracture status have not yet emerged, likely owing to confounding variables such as patient/donor age, tissue age (i.e, time since tissue formation), anatomic site, and differing experimental techniques. 
The purpose of this study is to compare the distributions of bone tissue properties in women with and without fragility fractures using FTIR imaging. We extend a prior study that examined the relationship of the mean FTIR properties to fragility fracture incidence [4] by (1) analyzing in detail the distributions of mineral and matrix properties in the bone tissue of biopsies from the fragility fracture and non-fracture cohorts and (2) analyzing biopsies from patients with and without prior histories of hormone replacement therapy (HRT) separately to distinguish the effects of HRT treatment history and fracture status. In addition to characterizing the heterogeneity of the tissue properties using techniques previously applied to FTIRI data $[13,18]$, we have also adapted methodology initially developed for analysis of the center, width, and tails of BMDD data assessed by qBEI [19], and applied it to FTIR imaging data. In this study we test the hypothesis that the bone tissue of patients with a history of fragility fracture is characterized by narrower distributions of mineral and matrix properties with attenuated tails shifted toward the center of the distribution, compared to that of patients with no history of fragility fracture.

\section{METHODS}

The biopsies analyzed in this study are a subset of those analyzed previously [4]. The original study included 54 iliac crest biopsies from women with low-trauma fractures $(+F x, n=32)$ and without fractures $(-F x, n=22)$. Patients with or without a history of treatment with estrogen-plus-progestin hormone replacement therapy (HRT) were included; patients with a history of treatment with all other bone-active agents (e.g., teriparatide, alendronate) were excluded. The following data was provided for each patient: code number, age at biopsy, HRT status $(\mathrm{yes} /$ no $=1 / 0)$, spine and hip BMD, T- 
score, and presence (1) or absence (0) of fractures at the time of biopsy. For the patients with fractures, biopsies were acquired 6 months- 5 years post-fracture. For each biopsy, three to nine cortical images and three to nine trabecular images from three $2-3-\mu \mathrm{m}$-thick sections were collected with an infrared imaging system (Spotlight 300, Perkin-Elmer Instruments, Waltham, MA, USA) [4].

Of the data from the original 54 biopsies, data from 33 were available for the current study ( $+F x n=21$, age: mean $54 \pm S D 15 y$; $-F x n=12$, age: $57.3 \pm 5.1$ y). Eight of the patients included in the -Fx group in the original study were taking HRT; all were included in the current study and analyzed separately from patients with no history of HRT (-Fx+HRT n=8, age: $58 \pm 5$ y; $-\mathrm{Fx}-\mathrm{HRT} \mathrm{n}=4$, age: $56 \pm 7$ y). Raw FTIR image data from the available 33 datasets were analyzed in a blinded fashion. Spectra were baseline corrected, and the PMMA spectral contribution was subtracted (ISys Chemical Imaging Analysis Software, Malvern Instruments Inc., Malvern, UK). The following FTIR parameters were calculated from the infrared spectrum collected at each pixel: the mineral:matrix ratio (area ratio of the phosphate $v_{1}$ and amide I peaks), the carbonate:phosphate ratio (area ratio of the carbonate $v_{2}$ and phosphate $v_{1}$ peaks), the collagen maturity (XLR, intensity ratio of the $1660 \mathrm{~cm}^{-1}$ and $1690 \mathrm{~cm}^{-1}$ bands), and the crystallinity (XST, intensity ratio of the $1030 \mathrm{~cm}^{-1}$ and $1020 \mathrm{~cm}^{-1}$ bands).

The analyses yielded spectroscopic images showing the spatial variation of each FTIR parameter within the sample (Fig. 1). For each image, four histograms were generated, each representing the set of bone pixel values associated with one of the four FTIR parameters. Each histogram was fit with a Gaussian curve. Four outcomes were used to characterize each FTIR parameter distribution: (1) the mean of the distribution to 
assess average composition, (2) the full width at half maximum (FHWM) of the Gaussian curve fit to the distribution to assess compositional heterogeneity at the center of the distribution, and (3) the $5^{\text {th }}$ percentile (low tail) and (4) the $95^{\text {th }}$ percentile (high tail) values of the distribution to assess the left and right tails of the distribution, respectively [19].

Statistical analyses were performed separately for cortical and trabecular bone. Linear mixed models were used to assess the relationship between groups (-Fx-HRT, Fx+HRT, and +Fx) and FTIR outcome measures while accounting for the repeated measurements made within each section (multiple FTIR images per section) and the multiple sections assessed per biopsy. Pairwise differences between groups were assessed with Tukey post-hoc tests. Differences in patient demographic and densitometric parameters (age, total hip BMD, lumbar spine BMD, hip t-score, spine t-score) among groups were assessed with Kruskal-Wallis tests. When significant, pairwise differences were assessed with Bonferroni-corrected pairwise Mann-Whitney U tests. Statistical analyses were performed in SAS version 9.3 (Cary, NC, USA), with a level of significance of 0.05 .

\section{RESULTS}

Demographic and densitometric data

The mean patient ages were similar across groups (Table 1). The lumbar spine BMD values were not different across groups, but the spine T scores differed across groups: the $-\mathrm{Fx}+\mathrm{HRT}$ group had a substantially greater mean $\mathrm{T}$ score compared to that of the $+\mathrm{Fx}$ group $(+206 \%, p=0.006)$ (Table 1). The trends observed in total hip BMD and total hip T 
score paralleled those noted for the spine $\mathrm{T}$ score, with the greatest values observed in the $-\mathrm{Fx}+\mathrm{HRT}$ group. Specifically, hip BMD and hip T score in the $-\mathrm{Fx}+\mathrm{HRT}$ group were respectively $37 \%$ and $318 \%$ greater than the corresponding values in the $+\mathrm{Fx}$ group (hip BMD $p=0.016$, hip $T$ score $p=0.025$ ) and trended toward $a+38 \%$ and $+274 \%$ difference vs. the $-\mathrm{Fx}-\mathrm{HRT}$ group (hip BMD $\mathrm{p}=0.057$, hip T score $\mathrm{p}=0.056$ ).

\section{Distribution means}

FTIR image data were first analyzed to determine the mean values of all four FTIR parameters. Across most of the FTIR parameters, the $-\mathrm{Fx}-\mathrm{HRT}$ group tended to have the smallest values, while the $-\mathrm{Fx}+\mathrm{HRT}$ and $+\mathrm{Fx}$ groups had larger values that were similar to each other (Fig. 2a). For mineral:matrix ratio, the trabecular tissue of $-\mathrm{Fx}-\mathrm{HRT}$ patients had a lower value than that of $-\mathrm{Fx}+\mathrm{HRT}$ patients $(-22 \%, \mathrm{p}<0.001)$ and $+\mathrm{Fx}$ patients $(-17 \%, \mathrm{p}=0.004)$. The cortical mineral:matrix values mirrored the trends in the trabecular values although the differences between groups did not reach statistical significance (Fig. 2a). Similar to the pattern in trabecular mineral:matrix ratio, the trabecular collagen maturity of tissue from $-\mathrm{Fx}-\mathrm{HRT}$ patients was lower than that of $-\mathrm{Fx}+$ HRT patients $(-18 \%, \mathrm{p}=0.006)$ and $+\mathrm{Fx}$ patients $(-18 \%, \mathrm{p}=0.002)$. Similarly, the cortical tissue of $-\mathrm{Fx}-\mathrm{HRT}$ patients trended toward lower collagen maturity than those of $-\mathrm{Fx}+\mathrm{HRT}$ patients and of $+\mathrm{Fx}$ patients $(-13 \%-\mathrm{Fx}-\mathrm{HRT}$ vs. $-\mathrm{Fx}+\mathrm{HRT}, \mathrm{p}=0.078 ;-13 \%-$ Fx-HRT vs. $+F x, p=0.058)$ (Fig. 2a). The mean crystallinity values were similar across groups, with the exception that trabecular crystallinity trended towards lower values in Fx-HRT patients versus + Fx patients $(-5 \%, p=0.071)$. Finally, for the carbonate:phosphate ratio, $-\mathrm{Fx}+\mathrm{HRT}$ patients had greater values relative to $+\mathrm{Fx}$ patients 
in trabecular tissue $(+29 \%, \mathrm{p}=0.032)$ and trended toward greater values relative to $+\mathrm{Fx}$ patients in cortical tissue $(+23 \%, \mathrm{p}=0.075)$. All other mean FTIR parameters were similar across groups.

\section{Distribution widths}

Next, FTIR image data were analyzed to assess the FWHM value of each FTIR parameter distribution. For the mineral:matrix ratio, trabecular tissue trended towards wider distributions in the $+\mathrm{Fx}$ group versus the $-\mathrm{Fx}+\mathrm{HRT}$ group $(+20 \%, \mathrm{p}=0.092)$ (Fig. $2 b)$. For the carbonate:phosphate ratio, trabecular distributions were widest in the $-\mathrm{Fx}-$ HRT group $(+121 \%$ vs. $-\mathrm{Fx}+\mathrm{HRT}, \mathrm{p}=0.028 ;+186 \%$ vs. $+\mathrm{Fx}, \mathrm{p}=0.004)$. The cortical carbonate:phosphate distributions were widest in the $-\mathrm{Fx}+\mathrm{HRT}$ group $(+414 \%$ vs. $-\mathrm{Fx}-$ HRT, $p=0.051 ;+280 \%$ vs. $+\mathrm{Fx}, \mathrm{p}=0.072$ ) although these trends did not reach statistical significance despite the large magnitudes of the differences due to the large variability in FWHM in the $-\mathrm{Fx}+\mathrm{HRT}$ and $+\mathrm{Fx}$ groups (Fig. 2). This trend was echoed in the cortical crystallinity distributions, which were wider in the $-\mathrm{Fx}+\mathrm{HRT}$ group than those in the - Fx-HRT group $(+39 \%, p=0.027)$ and those in the + Fx group $(+43 \%, p<0.001)($ Fig. $2 b)$. All other distribution widths were similar across groups.

\section{Distribution tails}

The $5^{\text {th }}$ and $95^{\text {th }}$ percentile values of the distribution of each FTIR parameter were calculated to characterize extreme low and high values, respectively, at the tails of the distributions (Fig. 2c,d). Across multiple FTIR parameters, the -Fx-HRT group tended to have smaller low-tail values than the $-\mathrm{Fx}+\mathrm{HRT}$ or $+\mathrm{Fx}$ groups, and these trends were 
more pronounced in the trabecular bone than in the cortical bone (Fig. 2c). Specifically, for the collagen maturity distributions, the trabecular bone of the -Fx-HRT group had smaller low-tail values than those in the $-\mathrm{Fx}+\mathrm{HRT}$ group $(-16 \%, \mathrm{p}=0.022)$ and in the $+\mathrm{Fx}$ group $(-17 \%, \mathrm{p}=0.005)$. The trabecular mineral:matrix and crystallinity distributions showed similar trends, exhibiting smaller low-tail values in the $-\mathrm{Fx}-\mathrm{HRT}$ vs. the $-\mathrm{Fx}+$ HRT group $(-19 \%, \mathrm{p}=0.058$ mineral:matrix; $-5 \%, \mathrm{p}<0.001$ crystallinity $)$ and vs. the +Fx group (-14\%, NS mineral:matrix; $-7 \%, \mathrm{p}<0.001$ crystallinity). In cortical bone, paralleling the pattern in the trabecular collagen maturity, the collagen maturity had smaller low-tail values in $-\mathrm{Fx}-\mathrm{HRT}$ vs. $-\mathrm{Fx}+\mathrm{HRT}(-26 \%$, p=0.0005) and vs. +Fx ($28 \%, \mathrm{p}<0.0001)$. The high-tail values differed primarily in the carbonate:phosphate ratio distributions, where the $-\mathrm{Fx}+\mathrm{HRT}$ group had greater values vs. $+\mathrm{Fx}(+26 \%, \mathrm{p}=0.032)$ and a similar trend vs. $-\mathrm{Fx}-\mathrm{HRT}(+54 \%, \mathrm{p}=0.071)$ in the cortical bone, whereas $-\mathrm{Fx}-\mathrm{HRT}$ had the greatest value $(+202 \%$, p $<0.001$ vs. $-\mathrm{Fx}+\mathrm{HRT} ;+292 \%$, p $<0.001$ vs. $+\mathrm{Fx})$ and $\mathrm{Fx}+\mathrm{HRT}$ had an intermediate value in trabecular bone $(+33 \%, \mathrm{p}=0.002$ vs. $+\mathrm{Fx})($ Fig. 1c, 2d). No other values at the tails of the distributions differed significantly across patient groups in trabecular or cortical bone.

\section{DISCUSSION}

The objective of this study was to compare the distributions of bone tissue properties in patients with and without fragility fractures using FTIR imaging. We hypothesized that the bone tissue of patients with a history of fragility fracture is characterized by narrower distributions of mineral and matrix properties with attenuated tails shifted toward the center of the distribution, compared to that of patients with no history of fragility fracture. 
The composition of iliac crest tissue differed in women with and without histories of fragility fractures and HRT. The trabecular properties showed the most distinct differences between groups, consistent with the greater expected metabolic activity in this tissue (Fig. 2a-d). As expected, - Fx-HRT patients had trabecular tissue with lower mean mineral content and collagen maturity compared to tissue in the $-\mathrm{Fx}+\mathrm{HRT}$ and $+\mathrm{Fx}$ groups. Bone tissue with greater mineral content is generally stronger and more brittle relative to less mineralized bone $[20,21]$, and cortical and trabecular bone with greater relative collagen maturity was previously associated with increased fracture risk [4]. In addition, in accord with our hypothesis, $-\mathrm{Fx}-\mathrm{HRT}$ patients had trabecular tissue with lower low-tail values of crystallinity and collagen maturity, compared to that in the $\mathrm{Fx}+\mathrm{HRT}$ and $+\mathrm{Fx}$ groups. However, the relative widths of the distributions of FTIR properties did not vary consistently across the groups. Finally, when tissue from HRTtreated and -untreated patients without fragility fractures was compared, the HRT-treated tissue had larger mean and low-tail values of mineral:matrix ratio and collagen maturity, consistent with greater tissue age and decreased osteoclastic resorption.

\section{Demographic and densitometric data}

Patients in the $-\mathrm{Fx}+\mathrm{HRT}$ group generally had a greater spine T score, hip T score, and hip BMD compared to patients in the $+\mathrm{Fx}$ group, with patients in the $-\mathrm{Fx}-\mathrm{HRT}$ group characterized by intermediate values. While the current study had a cross-sectional rather than a longitudinal design and cannot assess temporal changes in BMD within individuals, these findings of greater relative areal density in fracture-free patients treated with HRT compared to those with fractures, who were never treated with HRT, are 
broadly in accord with prior findings that estrogen-plus-progestin HRT increases BMD at the lumbar spine and the total hip [22].

\section{Role of altered collagen maturity distributions in fracture risk}

Of all the FTIR properties examined, the one that most consistently differed across groups in both cortical and trabecular bone was collagen maturity (Fig. 2). The differences in the collagen maturity distributions point to the presence of younger tissue in the $-\mathrm{Fx}-\mathrm{HRT}$ group, as compared to that in the $-\mathrm{Fx}+\mathrm{HRT}$ and $+\mathrm{Fx}$ groups.

The collagen maturity distributions of the trabecular bone were characterized by a smaller mean value in the $-\mathrm{Fx}-\mathrm{HRT}$ group relative to the other groups and no differences in the FWHMs, which, together with the smaller low-tail value in the -Fx-HRT group and no differences in the high-tail values, suggests that in the $-\mathrm{Fx}-\mathrm{HRT}$ group there is a shift toward less mature collagen on average, as well as toward an increased presence of the least mature collagen (represented by the low tail of the distribution) relative to the $\mathrm{Fx}+\mathrm{HRT}$ and $+\mathrm{Fx}$ groups. In cortical bone, the collagen maturity distributions showed analogous but less marked trends to those observed in the trabecular bone. The relatively greater proportions of immature collagen may reflect an increase in osteoblast activity or a decrease in resorption of new bone on trabecular surfaces in the $-\mathrm{Fx}-\mathrm{HRT}$ vs.

$-\mathrm{Fx}+\mathrm{HRT}$ and $+\mathrm{Fx}$ groups.

Variance in collagen maturity and other crosslink properties have been shown to be associated with bone mechanical properties and fracture resistance in multiple studies [4, 23-25]. In particular, greater mean collagen maturity in both cortical and trabecular bone is associated with increased fragility fracture risk [4]. Our study suggests that the 
low-tail values of the collagen maturity distribution, which represent the areas of the least mature collagen, especially distinguish tissue from HRT-naïve patients without fragility fractures as compared to HRT-treated patients without fragility fractures or patients with fractures.

\section{Role of altered mineral property distributions in fracture risk}

In agreement with the observed trends in collagen properties, the differences in the mineral:matrix ratio distributions point to the presence of younger tissue in the $-\mathrm{Fx}-\mathrm{HRT}$ group, as compared to that in the $-\mathrm{Fx}+\mathrm{HRT}$ and $+\mathrm{Fx}$ groups. In the mineral:matrix ratio, the primary differences in the trabecular distributions were observed in the mean values: trabecular bone of the $-\mathrm{Fx}-\mathrm{HRT}$ group had the lowest mean mineral content relative to the other groups.

Differences in crystallinity across groups were observed primarily at the tails of the distributions in both cortical and trabecular bone, although the $-\mathrm{Fx}-\mathrm{HRT}$ group trended toward having lower mean trabecular crystallinity than the + Fx group, consistent with the lower mean mineral:matrix ratio also observed in the $-\mathrm{Fx}-\mathrm{HRT}$ vs. the $+\mathrm{Fx}$ group. Because crystallinity is a measure of hydroxyapatite crystal size and stoichiometric perfection, which increase with tissue age [26], the 7\% smaller low-tail value in the cortical $-\mathrm{Fx}+\mathrm{HRT}$ group vs. the $+\mathrm{Fx}$ group suggests that the tissue from the -Fx+HRT patients included relatively more small, stoichiometrically imperfect crystals. The similar high-tail values across groups suggest that similar relative quantities of large, stoichiometric crystals were present in all groups. In trabecular bone, the low-tail value of the $-\mathrm{Fx}-\mathrm{HRT}$ group was $5 \%$ smaller than that of the $+\mathrm{Fx}$ group, and this difference was 
accompanied by trends toward a $5 \%$ smaller high-tail value vs. $+\mathrm{Fx}(\mathrm{p}=0.074)$, a $5 \%$ smaller mean value ( $\mathrm{p}=0.071)$, and no difference in FWHM, suggesting that the entire Fx-HRT distribution was shifted toward lower crystallinity values relative to that of the + Fx group. The shift toward lower values of trabecular crystallinity is congruent with the lower values of mineral:matrix ratio and collagen maturity observed in the $-\mathrm{Fx}+\mathrm{HRT}$ group, all of which are characteristic of younger tissue. The reduced quantity of relatively small crystals in the $+\mathrm{Fx}$ group relative to the $-\mathrm{Fx}+\mathrm{HRT}$ group is also consistent with reduced toughness and fracture resistance because loss of small crystals accompanies increased age-related fragility [27].

In the carbonate:phosphate ratio distributions, the $-\mathrm{Fx}+\mathrm{HRT}$ group had a greater mean value than the $+F x$ group in trabecular bone, with a similar trend in cortical bone $(+24 \%, p=0.075)$. Similarly, the $-\mathrm{Fx}+\mathrm{HRT}$ group had a greater high-tail value than the + Fx group, with a similar trend vs. $-\mathrm{Fx}-\mathrm{HRT}(+54 \%, \mathrm{p}=0.071)$, in cortical bone. Thus, tissue in the $-\mathrm{Fx}+\mathrm{HRT}$ group had the greatest mean values of carbonate substitution, and in cortical tissue this group also had the greatest high-tail values as well. Interpretation of this parameter is complex because it reflects variations in local availability of carbonate ions, but some trends are evident. In newly deposited bone mineral, carbonate substitution for phosphate increases with tissue age, but over time, as the mineral crystals grow larger and more perfect, carbonate substitution diminishes, and the carbonate:phosphate ratio may plateau [7, 28]. The larger carbonate:phosphate mean values in the $-\mathrm{Fx}+\mathrm{HRT}$ group vs. the $+\mathrm{Fx}$ group are consistent with older tissue age (See Comparison of HRT-treated and-untreated tissue). Trabecular carbonate:phosphate distributions were widest in the $-\mathrm{Fx}-\mathrm{HRT}$ group, which were also characterized by the 
greatest high-tail values; and cortical distributions trended toward being widest in the $\mathrm{Fx}+\mathrm{HRT}$ group, indicating that the greatest heterogeneity in carbonate substitution was in the non-fracture groups.

\section{Role of material heterogeneity in fracture risk}

The differences in distribution widths were observed in the three FTIR parameters that characterize mineral properties: mineral:matrix ratio, carbonate:phosphate ratio, and crystallinity. With the exception of a trend toward wider trabecular mineral:matrix distributions in the $+\mathrm{Fx}$ group versus the $-\mathrm{Fx}+\mathrm{HRT}$ group, the widest distributions were observed in tissue from patients without fragility fractures (Fig. 2B). In cortical crystallinity, the narrowest distribution was in the $+\mathrm{Fx}$ group. This trend is generally consistent with the results of previous studies using FTIR and qBEI showing narrower distributions of mineral properties in osteoporotic bone versus healthy bone $[5,19]$. Conversely, the distribution of the trabecular mineral:matrix ratio trended toward a wider distribution in the $+\mathrm{Fx}$ group than in the $-\mathrm{Fx}+\mathrm{HRT}$ group. Wider mineralization distributions in patients with fragility fractures versus controls were also observed using microradiography [17] and qBEI [19, 29]. However, a separate qBEI study of iliac crest trabeculae found the opposite trend [15]. Further investigation is required to explain these variable trends observed across studies.

The observed variability in the widths of the trabecular mineralization distributions with fracture status across studies may be explained in part by controlling for variations in turnover or tissue age. For example, when BMDDs from patients with and without fragility fractures were subdivided into low- and high-turnover groups, only 
the distribution of the non-fracture low-turnover subgroup was wider than that of the fracture group, while both low- and high-turnover fracture groups had similar BMDD widths [15]. Wider distributions are associated with low-turnover disease states and narrower distributions are associated with high-turnover disease states in studies employing qBEI and FTIRI $[15,30]$. The narrower distributions found in high-turnover biopsies suggest that in high-turnover disease states, resorption outpaces formation, leading to loss of newly formed tissue from bone surfaces, resulting in narrowed distributions of tissue material properties. Unfortunately, turnover data were not available for the patients in the current study, which precluded direct analysis of the effect of turnover on the observed distributions of FTIR properties.

\section{Implications of altered compositional distributions for fracture risk}

Changes in the distributions of mineral and matrix properties across groups suggest that the presence of newly formed as well as moderately mature tissue, is an important characteristic of healthy, fracture-resistant bone. The loss of smaller/less perfect crystals and immature collagen crosslinks in the $+\mathrm{Fx}$ and $-\mathrm{Fx}+\mathrm{HRT}$ groups vs. the $-\mathrm{Fx}-\mathrm{HRT}$ group accompanied a smaller FWHM of trabecular carbonate:phosphate ratio in the $+\mathrm{Fx}$ group relative to the $-\mathrm{Fx}-\mathrm{HRT}$ group. Together these data suggest that bone tissue in the + Fx group has a somewhat narrower range of these compositional properties than the other groups.

Heterogeneity of material properties below the length scale of $\sim 200 \mathrm{~nm}$ is expected to provide intrinsic toughening by promoting plasticity that resists crack initiation and propagation $[12,14]$; however, toughening mechanisms at multiple length 
scales are affected by osteoporosis [31], hindering isolation of the contribution of a single mechanism to fragility in experimental studies. Computational studies incorporating heterogeneous material properties at the nano- and microscales have predicted modest to substantial effects of material heterogeneity on macroscopic mechanical behavior, with the magnitude of the effects increasing as heterogeneity increases [12, 32, 33].

\section{Comparison of HRT-treated and-untreated tissue}

The differences in mean tissue properties between the $-\mathrm{Fx}+\mathrm{HRT}$ and $-\mathrm{Fx}-\mathrm{HRT}$ groups observed here are consistent with older tissue age in the HRT-treated tissue. Because our cross-sectional study was not designed to assess the effects of HRT on bone properties as a primary outcome, the differences in mean tissue properties between the HRT-treated and -untreated groups may also reflect the contribution of underlying differences in bone properties between the patient groups. Nevertheless, our data are in accord with trends observed in previous longitudinal studies of iliac crest biopsies of postmenopausal women treated with HRT. Increased collagen maturity was observed in a biochemical analysis of biopsies from women treated for 1 year with estradiol HRT [34], and increased mean mineralization was observed in a microradiographic study of biopsies from women treated for 2 years with estrogen-plus-progestin HRT [35]. Similarly, in an FTIR imaging study of double iliac crest biopsies from postmenopausal women aged 4555 years receiving 2 years estrogen-plus-progestin HRT, trabeculae exhibited increased mineral:matrix ratio $(+102 \%)$, collagen maturity $(+41 \%)$, and crystallinity $(+28 \%)[36]$. In the current study, the differences in properties between the $-\mathrm{Fx}+\mathrm{HRT}$ and $-\mathrm{Fx}-\mathrm{HRT}$ groups were analogous but smaller in magnitude $(+28 \%$ mineral:matrix, $+22 \%$ collagen 
maturity, $+3 \%$ (NS) crystallinity). The larger mean values of mineral:matrix ratio and collagen maturity noted in our HRT-treated biopsies are consistent with greater tissue age, and with the decreased osteoclastic resorption observed in a prior histomorphometric analyses of double biopsies from HRT-treated patients [37, 38].

In addition, consistent with reduced resorption, the greater mean values of the FTIR properties in the $-\mathrm{Fx}+\mathrm{HRT}$ tissue versus the $-\mathrm{Fx}-\mathrm{HRT}$ trabeculae were accompanied by greater low-tail values, reflecting a relative paucity of tissue with the lowest mineral content, smallest/least perfect crystals, and least mature collagen. In accordance with prior microradiographic analyses [35], the widths of the trabecular mineral:matrix distributions were similar in the $-\mathrm{Fx}-\mathrm{HRT}$ and $-\mathrm{Fx}+\mathrm{HRT}$ groups; but the carbonate:phosphate distributions were narrower in the $-\mathrm{Fx}+\mathrm{HRT}$ group compared to the -Fx-HRT group, suggesting that there were minimal differences between groups in overall trabecular compositional heterogeneity in mineralization but that the $-\mathrm{Fx}+\mathrm{HRT}$ group had less heterogeneous carbonate substitution. These observations are consistent with the expected effects of an agent that reduces osteoclastic activity, thereby reducing young tissue and allowing the composition of existing tissue to become more uniform by reducing remodeling. However, cortical tissue of the $-\mathrm{Fx}+\mathrm{HRT}$ group had wider crystallinity distributions than either group with no history of HRT treatment, perhaps more consistent the with the anabolic effect of high doses of HRT previously observed in cortical bone [39]. Thus, decreased osteoclastic resorption with HRT may allow existing tissue to reach older tissue ages prior to resorption, shifting the material property distributions to higher mean values of mineral:matrix ratio and collagen maturity and 
increasing the low-tail values of the distributions, thereby contributing to the increased BMD observed with HRT [22].

\section{Strengths, limitations, and conclusions}

The study design contributes to some inherent limitations and strengths of the current study. One limitation is that we retrospectively reanalyzed existing FTIR data from historical biopsies because the original biopsies were not available to rescan. The data files from some patients in the original study [4] were excluded because they were not suitable for analysis of FWHM or high- and low-tail values, and the excluded files were disproportionately in the $-\mathrm{Fx}$ groups. The exclusions resulted in greater numbers of patients in the $+F x$ group than in the $-F x$ groups. Furthermore, the observed differences in compositional properties between the $-\mathrm{Fx}+\mathrm{HRT}$ and $-\mathrm{Fx}-\mathrm{HRT}$ groups prevented pooling of these groups to maximize statistical power in a pooled -Fx group. The statistical power was nevertheless sufficient to detect differences in mean, FWHM, and high- and low-tail values of the FTIR parameter distributions between groups. However, the power was insufficient to perform a multiple logistic regression to explore the associations between the 16 compositional variables (mean, FWHM, high-tail, and lowtail value of each of the 4 FTIR parameters), BMD, and fracture incidence. In addition, the small size of the $-\mathrm{Fx}-\mathrm{HRT}$ group prevented implementation of an unequal-variances model for the carbonate:phosphate ratio. A second limitation of the observational, retrospective study design is that turnover and menopausal status were not assessed for the patients in the current study. Thus, while the mean patient ages at biopsy did not differ across groups, the extent to which underlying differences in turnover contributed to 
variability in FTIR properties within or between groups could not be quantified. Finally, HRT treatment was recorded only as a binary variable $(0,1)$; thus analysis of relationships in FTIR outcome variables with HRT treatment duration was not possible.

Our study is advantageous because tissue properties were analyzed by FTIRI, a technique with outcomes previously validated using independent techniques $[5,8,9]$ and shown to contribute to fragility fracture independently of BMD [4]. In contrast to prior studies, which averaged FTIR properties across multiple images per specimen to generate average values for each biopsy $[4,13]$, the current study used a repeated-measures analysis, which took advantage of the collection of multiple images within each specimen each biopsy, took into account the variability within each image, and provided a better overall estimate of the true variability in FTIR properties within the biopsy. Finally, biopsies were taken from living human patients with and without known histories of fragility fractures. In living humans, comparison of bone tissue properties in cohorts of patients with and without fragility fractures remains the only experimental approach that enables tissue composition to be related to whole-bone structural behavior.

In conclusion, the distributions of mineral and collagen properties differ between patients with and without fragility fractures. Not only the mean values of the mineral and matrix properties, but also the low-tail values, which represent newly formed tissue, were altered in patients with fragility fractures compared to those without fragility fractures. This suggests that, in addition to mean compositional properties, the extreme values of the tissue properties are important indicators of healthy, fracture-resistant bone. 


\section{DISCLOSURES}

All authors state that they have no conflicts of interest.

\section{ACKNOWLEDGMENTS}

This work was supported in part by an American Society for Bone and Mineral Research

Junior Faculty Osteoporosis Research Award and Cornell Engineering Learning

Initiatives. This material is based upon work supported by the National Science

Foundation Graduate Research Fellowship Program under Grant No. NSF DGE-

1144153. We thank Dr. Adele Boskey for the raw data and for helpful discussions. 


\section{REFERENCES}

[1] Siris ES, Brenneman SK, Miller PD, Barrett-Connor E, Chen YT, Sherwood LM, et al. Predictive value of low BMD for 1-year fracture outcomes is similar for postmenopausal women ages 50-64 and 65 and Older: results from the National Osteoporosis Risk Assessment (NORA). J Bone Miner Res 2004;19:1215-20.

[2] Hillier TA, Stone KL, Bauer DC, Rizzo JH, Pedula KL, Cauley JA, et al. Evaluating the value of repeat bone mineral density measurement and prediction of fractures in older women: the study of osteoporotic fractures. Archives of internal medicine 2007;167:15560.

[3] Donnelly E, Lane JM, Boskey AL. Research perspectives: The 2013 AAOS/ORS research symposium on Bone Quality and Fracture Prevention. J Orthop Res 2014;32:855-64.

[4] Gourion-Arsiquaud S, Faibish D, Myers E, Spevak L, Compston J, Hodsman A, et al. Use of FTIR spectroscopic imaging to identify parameters associated with fragility fracture. J Bone Miner Res 2009;24:1565-71.

[5] Boskey A, Mendelsohn R. Infrared analysis of bone in health and disease. J Biomed Opt 2005;10:031102.

[6] Faibish D, Gomes A, Boivin G, Binderman I, Boskey A. Infrared imaging of calcified tissue in bone biopsies from adults with osteomalacia. Bone 2005;36:6-12.

[7] Ou-Yang H, Paschalis EP, Mayo WE, Boskey AL, Mendelsohn R. Infrared microscopic imaging of bone: spatial distribution of CO3(2-). J Bone Miner Res 2001;16:893-900.

[8] Paschalis EP, Verdelis K, Doty SB, Boskey AL, Mendelsohn R, Yamauchi M. Spectroscopic characterization of collagen cross-links in bone. J Bone Miner Res 2001;16:1821-8.

[9] Pleshko N, Boskey A, Mendelsohn R. Novel infrared spectroscopic method for the determination of crystallinity of hydroxyapatite minerals. Biophys J 1991;60:786-93. [10] Renders GA, Mulder L, Langenbach GE, van Ruijven LJ, van Eijden TM. Biomechanical effect of mineral heterogeneity in trabecular bone. J Biomech 2008;41:2793-8.

[11] Ruffoni D, Fratzl P, Roschger P, Klaushofer K, Weinkamer R. The bone mineralization density distribution as a fingerprint of the mineralization process. Bone 2007;40:1308-19.

[12] Tai K, Dao M, Suresh S, Palazoglu A, Ortiz C. Nanoscale heterogeneity promotes energy dissipation in bone. Nat Mater 2007;6:454-62.

[13] Donnelly E, Meredith DS, Nguyen JT, Gladnick BP, Rebolledo BJ, Shaffer AD, et al. Reduced cortical bone compositional heterogeneity with bisphosphonate treatment in postmenopausal women with intertrochanteric and subtrochanteric fractures. J Bone Miner Res 2012;27:672-8.

[14] Ritchie RO. The conflicts between strength and toughness. Nat Mater 2011;10:81722.

[15] Ciarelli TE, Tjhia C, Rao DS, Qiu S, Parfitt AM, Fyhrie DP. Trabecular packet-level lamellar density patterns differ by fracture status and bone formation rate in white females. Bone 2009;45:903-8. 
[16] Gourion-Arsiquaud S, Lukashova L, Power J, Loveridge N, Reeve J, Boskey AL. Fourier transform infrared imaging of femoral neck bone: reduced heterogeneity of mineral-to-matrix and carbonate-to-phosphate and more variable crystallinity in treatment-naive fracture cases compared with fracture-free controls. Journal of bone and mineral research : the official journal of the American Society for Bone and Mineral Research 2013;28:150-61.

[17] Bousson V, Bergot C, Wu Y, Jolivet E, Zhou LQ, Laredo JD. Greater tissue mineralization heterogeneity in femoral neck cortex from hip-fractured females than controls. A microradiographic study. Bone 2011;48:1252-9.

[18] Gourion-Arsiquaud S, Allen MR, Burr DB, Vashishth D, Tang SY, Boskey AL. Bisphosphonate treatment modifies canine bone mineral and matrix properties and their heterogeneity. Bone 2010;46:666-72.

[19] Roschger P, Paschalis EP, Fratzl P, Klaushofer K. Bone mineralization density distribution in health and disease. Bone 2008;42:456-66.

[20] Currey JD. The mechanical consequences of variation in the mineral content of bone. J Biomech 1969;2:1-11.

[21] Currey JD. Tensile yield in compact bone is determined by strain, post-yield behaviour by mineral content. J Biomech 2004;37:549-56.

[22] Cauley JA, Robbins J, Chen Z, Cummings SR, Jackson RD, LaCroix AZ, et al. Effects of estrogen plus progestin on risk of fracture and bone mineral density: the Women's Health Initiative randomized trial. JAMA 2003;290:1729-38.

[23] Vashishth D. The role of the collagen matrix in skeletal fragility. Curr Osteoporos Rep 2007;5:62-6.

[24] Saito M, Mori S, Mashiba T, Komatsubara S, Marumo K. Collagen maturity, glycation induced-pentosidine, and mineralization are increased following 3-year treatment with incadronate in dogs. Osteoporos Int 2008;19:1343-54.

[25] Currey JD. Role of collagen and other organics in the mechanical properties of bone. Osteoporos Int 2003;14:S29-S36.

[26] Gourion-Arsiquaud S, Burket JC, Havill LM, DiCarlo E, Doty SB, Mendelsohn R, et al. Spatial variation in osteonal bone properties relative to tissue and animal age. J Bone Miner Res 2009;24:1271-81.

[27] Chatterji S, Wall JC, Jeffery JW. Age-related changes in the orientation and particle size of the mineral phase in human femoral cortical bone. Calcif Tissue Int 1981;33:56774.

[28] Tarnowski CP, Ignelzi MA, Jr., Morris MD. Mineralization of developing mouse calvaria as revealed by Raman microspectroscopy. J Bone Miner Res 2002;17:1118-26. [29] Busse B, Hahn M, Soltau M, Zustin J, Puschel K, Duda GN, et al. Increased calcium content and inhomogeneity of mineralization render bone toughness in osteoporosis: mineralization, morphology and biomechanics of human single trabeculae. Bone 2009;45:1034-43.

[30] Boskey AL, DiCarlo E, Paschalis E, West P, Mendelsohn R. Comparison of mineral quality and quantity in iliac crest biopsies from high- and low-turnover osteoporosis: an FT-IR microspectroscopic investigation. Osteoporos Int 2005;16:2031-8.

[31] Thurner PJ, Katsamenis OL. The role of nanoscale toughening mechanisms in osteoporosis. Curr Osteoporos Rep 2014;12:351-6. 
[32] van der Linden JC, Birkenhager-Frenkel DH, Verhaar JA, Weinans H. Trabecular bone's mechanical properties are affected by its non-uniform mineral distribution. Journal of biomechanics 2001;34:1573-80.

[33] Bourne BC, van der Meulen MC. Finite element models predict cancellous apparent modulus when tissue modulus is scaled from specimen CT-attenuation. J Biomech 2004;37:613-21.

[34] Holland EF, Studd JW, Mansell JP, Leather AT, Bailey AJ. Changes in collagen composition and cross-links in bone and skin of osteoporotic postmenopausal women treated with percutaneous estradiol implants. Obstetrics and gynecology 1994;83:180-3. [35] Boivin G, Vedi S, Purdie DW, Compston JE, Meunier PJ. Influence of estrogen therapy at conventional and high doses on the degree of mineralization of iliac bone tissue: a quantitative microradiographic analysis in postmenopausal women. Bone 2005;36:562-7.

[36] Paschalis EP, Boskey AL, Kassem M, Eriksen EF. Effect of hormone replacement therapy on bone quality in early postmenopausal women. J Bone Miner Res 2003;18:9559.

[37] Eriksen EF, Langdahl B, Vesterby A, Rungby J, Kassem M. Hormone replacement therapy prevents osteoclastic hyperactivity: A histomorphometric study in early postmenopausal women. J Bone Miner Res 1999;14:1217-21.

[38] Holland EF, Chow JW, Studd JW, Leather AT, Chambers TJ. Histomorphometric changes in the skeleton of postmenopausal women with low bone mineral density treated with percutaneous estradiol implants. Obstetrics and gynecology 1994;83:387-91.

[39] Vedi S, Bell KL, Loveridge N, Garrahan N, Purdie DW, Compston JE. The effects of hormone replacement therapy on cortical bone in postmenopausal women. A histomorphometric study. Bone 2003;33:330-4. 


\section{FIGURE LEGENDS}

Figure 1. Representative examples of pixel histograms generated from single FTIR images (insets) for each parameter and group with Gaussian fits, of trabecular (a) mineral:matrix ratio, (b) collagen maturity, (c) carbonate:phosphate ratio, (d) crystallinity for patients with fragility fractures $(+\mathrm{Fx})$ or without fragility fractures, with or without hormone replacement therapy ( $-\mathrm{Fx}+\mathrm{HRT}$ or $-\mathrm{Fx}-\mathrm{HRT}$, respectively). The mean, full width at half maximum (FWHM), low-tail ( $5^{\text {th }}$ percentile), and high-tail $\left(95^{\text {th }}\right.$ percentile) values of the Gaussian curves are indicated on each histogram. Scale bars on FTIR images $=50 \mu \mathrm{m}$

Figure 2. Cortical and trabecular FTIR properties reported as image pixel distribution (a) mean, (b) full width at half maximum (FWHM), (c) $5^{\text {th }}$ percentile value, and (d) $95^{\text {th }}$ percentile value for patients with fragility fractures $(+\mathrm{Fx})$ or without fragility fractures, with or without hormone replacement therapy $\left(-\mathrm{Fx}+\mathrm{HRT}\right.$ or $-\mathrm{Fx}-\mathrm{HRT}$, respectively); ${ }^{*} \mathrm{p}$ $<0.05, \# p<0.1$. Bar heights and error bars indicate raw mean and 95\% confidence intervals, respectively; p-values reflect adjustment for multivariate repeated measures. 


\section{TABLES}

Table 1. Patient demographic and densitometric characteristics reported as means with $95 \%$ confidence intervals in brackets. Bold text highlights pairs of values with a significant difference between groups; ${ }^{a} \mathrm{p}<0.05,{ }^{\mathrm{aa}} \mathrm{p}<0.1$ vs. $-\mathrm{Fx}-\mathrm{HRT} ;{ }^{\mathrm{b}} \mathrm{p}<0.05,{ }^{\mathrm{bb}} \mathrm{p}<$ 0.1 vs. - Fx + HRT by Kruskal-Wallis with Bonferroni-corrected pairwise Mann-Whitney post-hoc.

\begin{tabular}{|c|c|c|c|c|}
\hline & $+\mathbf{F x}$ & $-F x-H R T$ & $-\mathbf{F x}+$ HRT & $\begin{array}{c}\text { Kruskal-Wallis } \\
\text { p-value }\end{array}$ \\
\hline Age at Biopsy & 54 & 56 & 58 & 0.822 \\
\hline (y) & {$[46,62]$} & {$[44,67]$} & {$[54,62]$} & \\
\hline Spine BMD & 0.78 & 0.96 & 0.81 & 0.302 \\
\hline$\left(\mathrm{g} / \mathrm{cm}^{2}\right)$ & {$[0.70,0.87]$} & {$[0.61,1.30]$} & {$[0.64,0.98]$} & \\
\hline Spine BMD T Score & $\begin{array}{l}-1.9^{b} \\
{[-2.5,-1.3]}\end{array}$ & $\begin{array}{l}-1.0 \\
{[-3.9,1.9]}\end{array}$ & $\begin{array}{l}1.8 \\
{[-0.2,3.8]}\end{array}$ & 0.007 \\
\hline $\begin{array}{l}\text { Hip BMD } \\
\left(\mathrm{g} / \mathrm{cm}^{2}\right)\end{array}$ & $\begin{array}{l}0.70^{b} \\
{[0.54,0.87]}\end{array}$ & $\begin{array}{l}0.70^{\mathbf{b b}} \\
{[0.63,0.76]}\end{array}$ & $\begin{array}{l}1.12 \\
{[0.97,1.27]}\end{array}$ & 0.005 \\
\hline Hip BMD T Score & $\begin{array}{l}-2.5^{b} \\
{[-3.4,-1.6]}\end{array}$ & $\begin{array}{l}-2.0^{\mathbf{b b}} \\
{[-2.7,-1.3]}\end{array}$ & $\begin{array}{l}1.2 \\
{[-0.1,2.4]}\end{array}$ & 0.004 \\
\hline
\end{tabular}




\section{FIGURES}

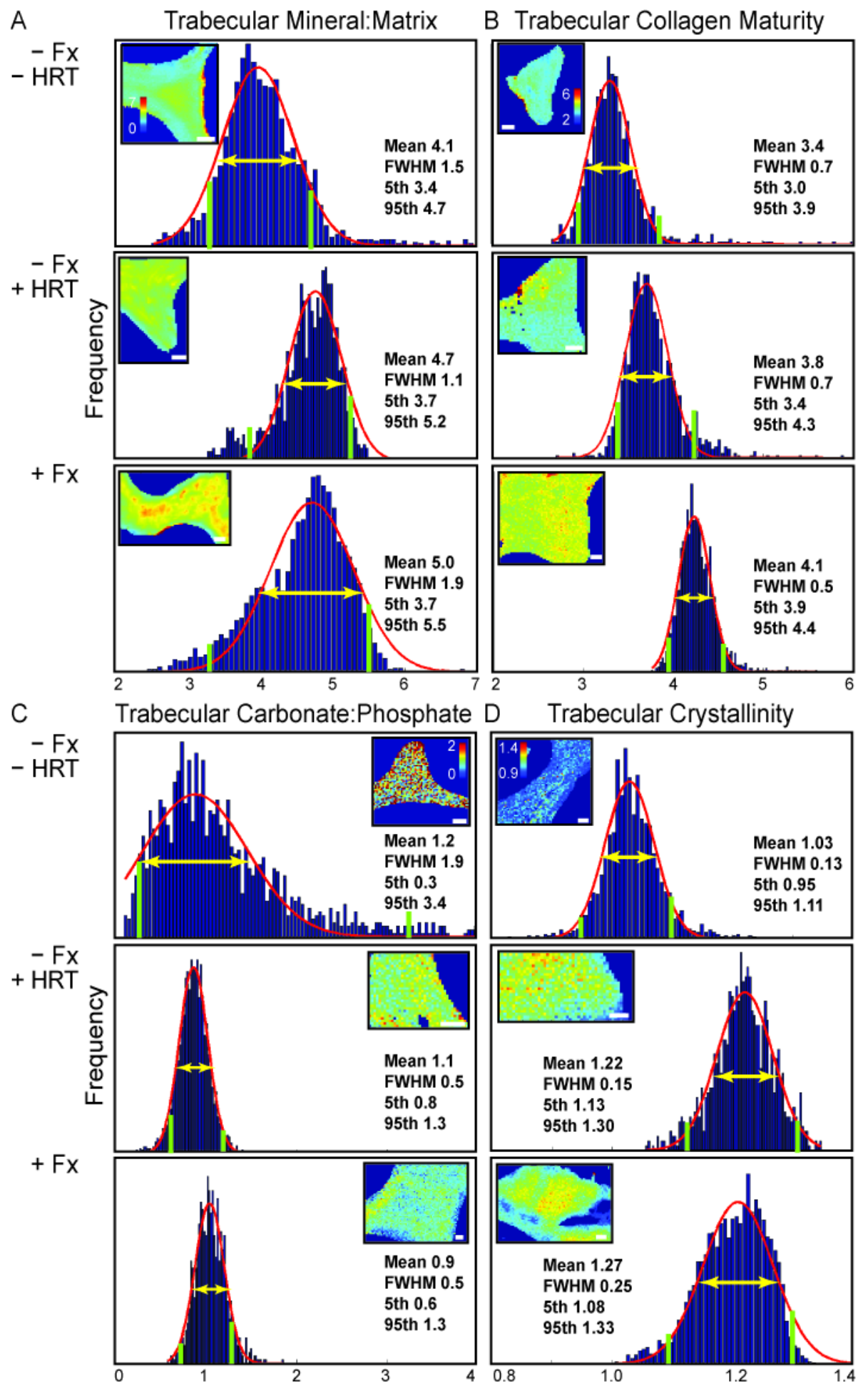

Figure 1. Representative examples of pixel histograms generated from single FTIR images (insets) for each parameter and group with Gaussian fits, of trabecular (a) 
mineral:matrix ratio, (b) collagen maturity, (c) carbonate:phosphate ratio, (d) crystallinity for patients with fragility fractures $(+\mathrm{Fx})$ or without fragility fractures, with or without hormone replacement therapy $(-\mathrm{Fx}+\mathrm{HRT}$ or $-\mathrm{Fx}-\mathrm{HRT}$, respectively). The mean, full width at half maximum (FWHM), low-tail ( $5^{\text {th }}$ percentile), and high-tail $\left(95^{\text {th }}\right.$ percentile) values of the Gaussian curves are indicated on each histogram. Scale bars on FTIR images $=50 \mu \mathrm{m}$. 

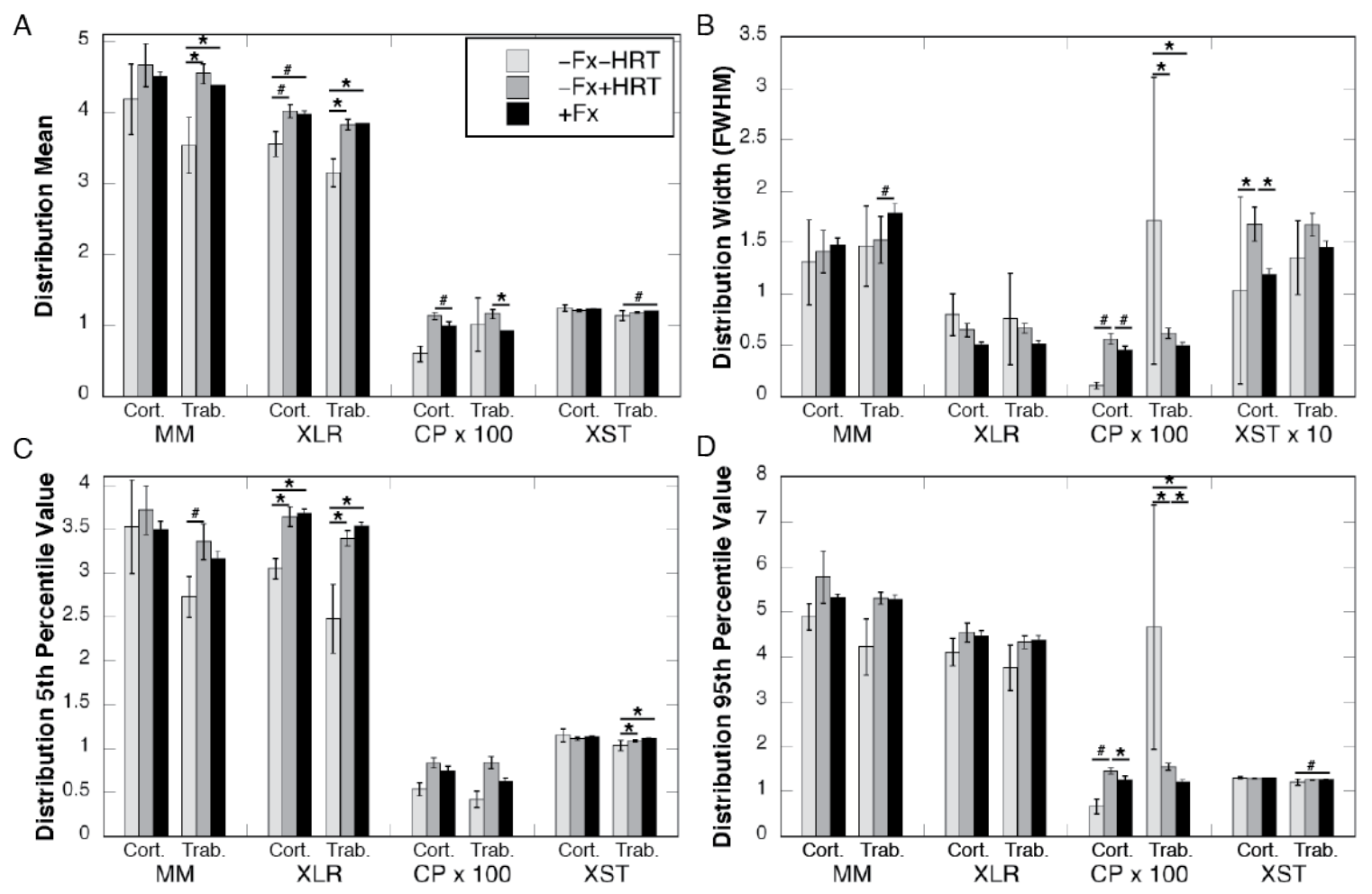

Figure 2. Cortical and trabecular FTIR properties reported as image pixel distribution (a) mean, (b) full width at half maximum (FWHM), (c) $5^{\text {th }}$ percentile value, and (d) $95^{\text {th }}$ percentile value for patients with fragility fractures $(+\mathrm{Fx})$ or without fragility fractures, with or without hormone replacement therapy $\left(-\mathrm{Fx}+\mathrm{HRT}\right.$ or $-\mathrm{Fx}-\mathrm{HRT}$, respectively); ${ }^{*} \mathrm{p}$ $<0.05, \# \mathrm{p}<0.1$. Bar heights and error bars indicate raw mean and 95\% confidence intervals, respectively; p-values reflect adjustment for multivariate repeated measures. 